\title{
Differentiating the stiffness of the side of the car
}

\author{
Stanislav Evtukov ${ }^{1}$, Yaroslav Vasilev ${ }^{1}$, Vseslav Voronin ${ }^{1}$, and Egor Golov ${ }^{{ }^{*}}$ \\ ${ }^{1}$ Saint Petersburg State University of Architecture and Civil Engineering, 4 2-nd Krasnoarmeiskaya \\ ul., Saint Petersburg, Russia, 190005
}

\begin{abstract}
Expertise on the materials of road accidents (road accidents), calculate the speed of the vehicles involved in the accident by analyzing the resulting deformation damage by the average stiffness coefficients of the vehicle bodies. But this entails the presence of an error in the calculation and, as a result, not a correct result due to the fact that the internal structure of the side of the car is heterogeneous - the stiffness in each of its areas is different. To eliminate possible errors when using the averaged coefficients, the article offers a method for calculating the stiffness coefficients for various areas of the side of cars that have received deformations as a result of an accident.
\end{abstract}

When cars collide, elastic and plastic deformations occur, the magnitude and nature of which depend on the relative velocity, the direction of impact, the rigidity of the structure and its mass, the density and hardening of their materials, the area of deformation and other factors. Experts-car technicians under "deformation" denote the presence of a change in the shape of the vehicle - a dent, bend, metal extraction, etc. In the mechanics of a deformable solid, deformation is a component of the strain tensor, a measure of the shape of a body at a point and its small neighborhood. Deformation can also be a measure of the shape of an object, if it is deformed uniformly. The deformation of the vehicle can be obtained as a result of a collision or impact of the vehicle with another object (for example, another vehicle, fence, etc.).

The study of the process of collision of a vehicle is based on the physical theory of impact of solid bodies. The theory of impact, during its four hundred years of development, has been mainly concerned with the study of the impact effect. According to the research of numerous authors, at present, when determining the kinematic, force and energy parameters of the collision of objects (the time of impact, the force of interaction, etc.), satisfactory results are obtained only experimentally. At the same time, the well-known models of the physical theory of impact do not always allow us to obtain correct calculation results when analyzing the pair interaction of such complex objects as vehicles.

From the point of view of the physical model, the impact is considered as a short-term process of interaction of bodies that proceeds with certain kinematic, force and energy parameters, the values of which can be calculated. In general, in theoretical mechanics, a shock is a process of interaction of bodies, in which for an infinitesimal period of time, the velocities change to a finite value.

* Corresponding author: egorgoloff@yandex.ru 
The collision intensity is estimated based on the strain energy of the damaged PBX. The strain energy is calculated from the results of collisions. A significant result of the research is that the deforming force increases approximately linearly with increasing strain - hence, the strain energy can be calculated from the value of the residual strain.

The energy of the PBX deformation during the collision is equivalent to the corresponding change in the kinetic energy. In a collision with a concrete barrier with $100 \%$ overlap, or in collisions after which the PBX has an insignificant reserve of kinetic energy, it can be assumed that the kinetic energy possessed by the vehicle before the collision is equal to the deformation energy [1].

The greatest difficulty in studying the collision of a vehicle, in contrast to the study of the impact of homogeneous solids, is caused by the fact that the vehicle is an anisotropic body that has different physical properties in different sections and consists of a variety of media (elements, parts, assemblies and aggregates), sharply limited by the spatial, strength and other configurations of materials from other, touching and/or interacting media. The anisotropy of the vehicle determines the nonlinear nature of the dependencies between stress and strain, speed and strain, etc. when cars collide. With a small impact force, the vehicles behave like elastic bodies, with a significant one-irreversible deformations (damage) occur. In both cases, complex wave and thermal processes also occur during the deformations of cars $[2,3,4,5]$.

When determining the actual speed of a car at the time of an accident based on residual deformations, experts use 2 main methods of calculation: finite element analysis and the CRASH3 algorithm. The finite element method is quite complex and requires an expert not only to know its theory, but also to be able to perform complex modeling of processes in finite element software, which is also expensive and not always available. In this connection, the CRASH3 algorithm is successfully used by most specialists, due to its simplicity of calculation and the permissible error $[6,7,8]$.

However, the essence of this method is that an insignificant error is achieved only when investigating an accident with the presence of large, significant deformations - this is due to the fact that the calculation uses the average value of deformations (as well as stiffness coefficients) and the dependence of the energy consumption for its development on the square of the speed. This phenomenon is clearly demonstrated by the graph in Figure 1.

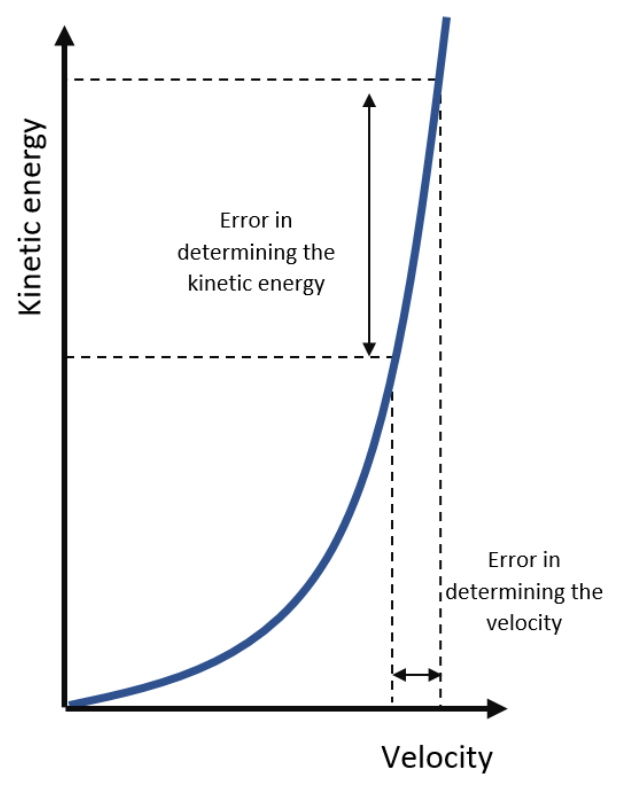

Fig. 1. Dependence of errors in the calculation of kinetic energy and velocity. 
But on the roads there are accidents with minor, local deformations, and in such situations, it is unacceptable to use the average values of the stiffness and deformation coefficients due to the fact that this will lead to a significant error in determining the collision speed. Also, the results of the calculation depend on the rigidity of the car body, which is characterized by coefficients - the higher their values, the greater the rigidity of the car and to achieve the resulting deformations, a large energy is required, and therefore the collision speed.

Previously, the dependence of the stiffness coefficients of the front part of the car on the place of application of force was established, in this article the rigidity of the side part of the car will be established. To differentiate the lateral rigidity of the car vertically, it is necessary to examine the crash test for a collision with a pole, and vertically - with a cart.

A number of similar crash tests were analyzed, differing only in the make and model of the participating car, let's consider the results of research on the example of NHTSA crash test No. 10780[9], where in a staged accident, a Jeep Grand Cherokee (weight - $2306 \mathrm{~kg}$ ) was hit by a laboratory cart MDB (Moving deformable barrier) (weight $1366 \mathrm{~kg}$ ) at a speed of $62.07 \mathrm{~km} / \mathrm{h}$ at an angle of $27^{\circ}$. Figures 2-3 and 4-5 show the car and cart before and after the collision, respectively.

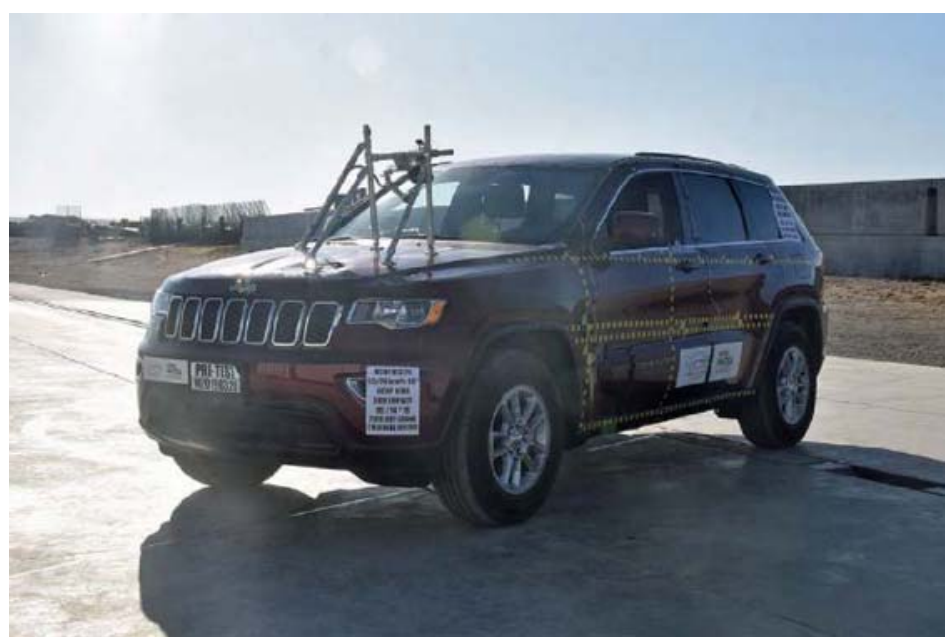

Fig.2. Jeep Grand Cherokee before the collision.

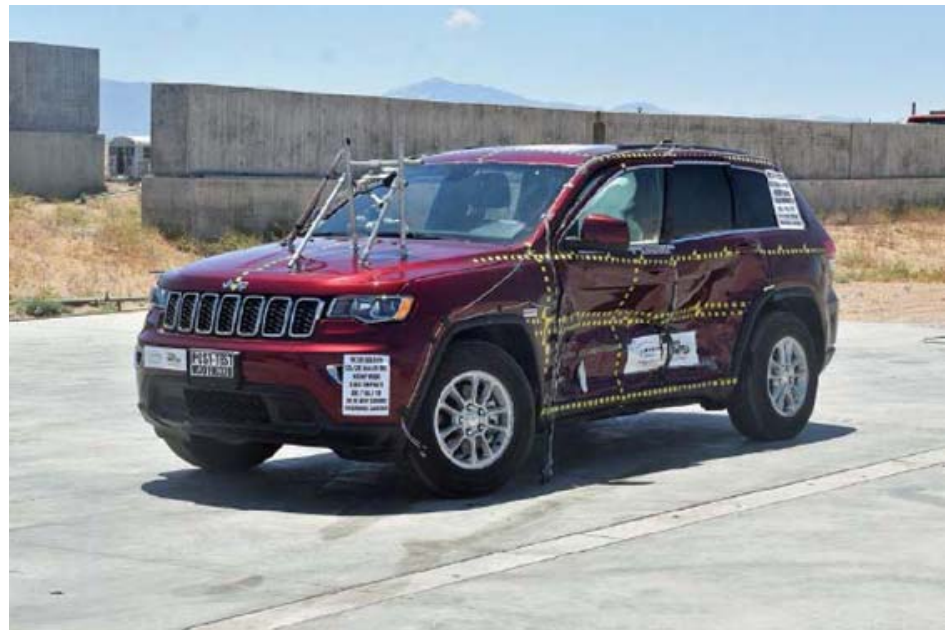

Fig. 3. Jeep Grand Cherokee car after collision. 


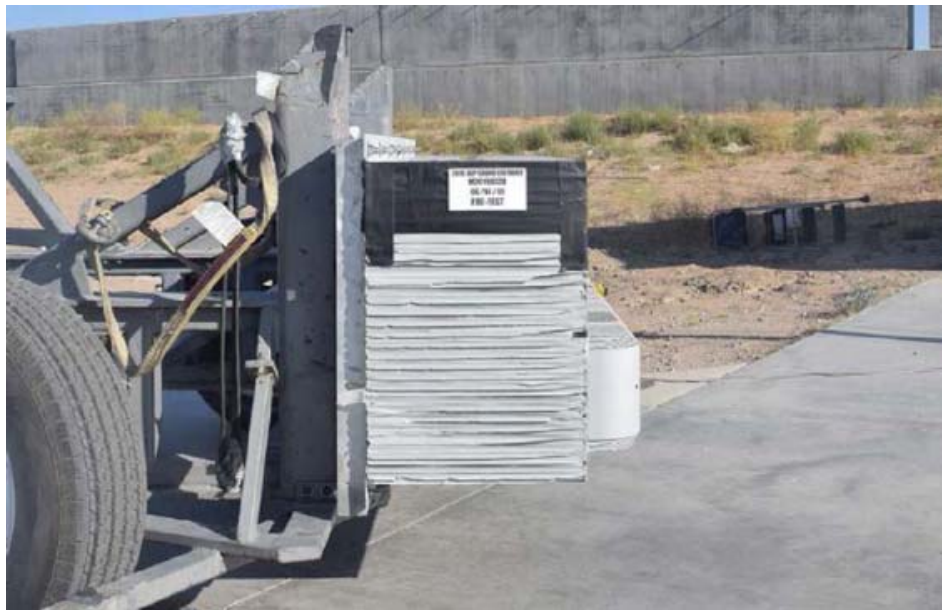

Fig. 4. MDB trolley before collision.

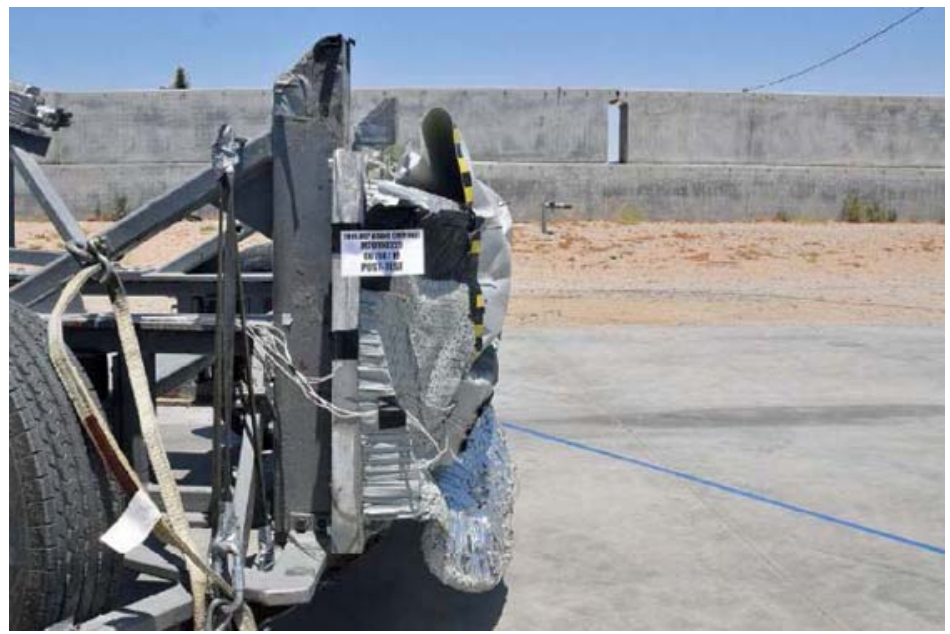

Fig. 5. MDB trolley after collision.

Table 1 shows the results of measuring the deformations of the Jeep Grand Cherokee and the MDB bogie in accordance with the designations shown in Figures 6 and 7.

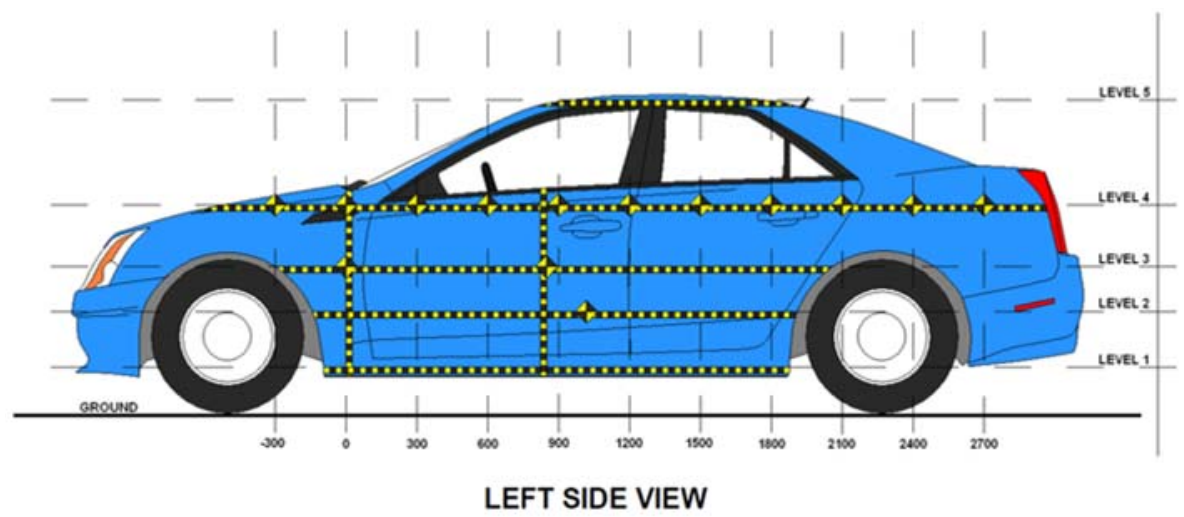

Fig. 6. Marking of deformations on the Jeep Grand Cherokee. 


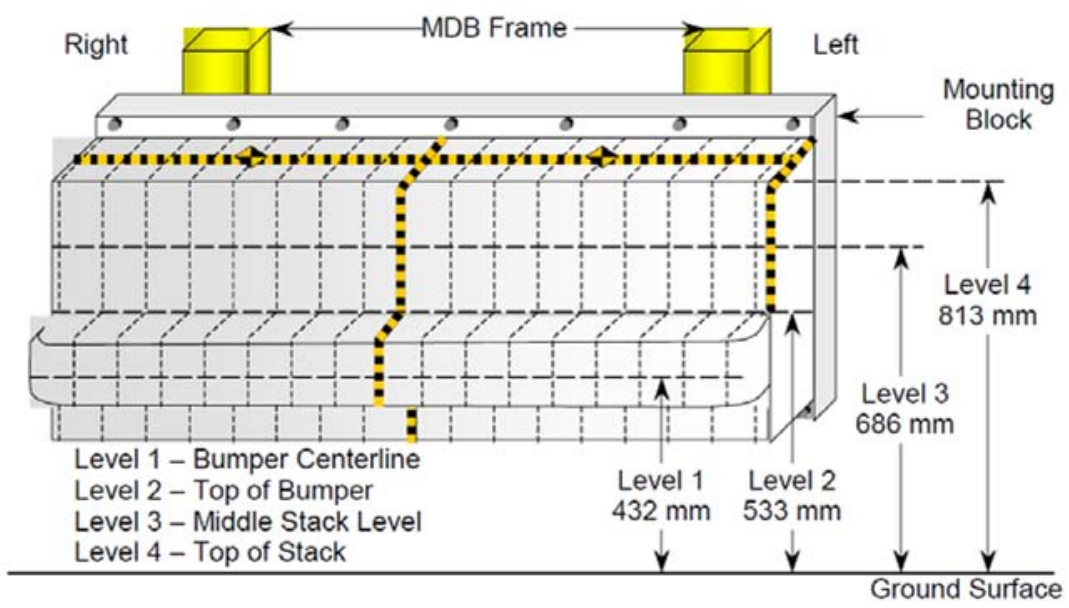

Fig. 7. Designation of deformations on MDB trolleys.

Table 1. Results of measuring the deformations of the Jeep Grand Cherokee and the MDB truck.

\begin{tabular}{|c|c|c|c|c|c|c|c|c|c|c|c|}
\hline \multicolumn{6}{|c|}{ Deformation of the Jeep Grand Cherokee car } & \multicolumn{6}{|c|}{ MDB Trolley deformation } \\
\hline $\begin{array}{l}\text { Level/ } \\
\text { points }\end{array}$ & 1 & 2 & 3 & 4 & 5 & & $\begin{array}{l}\text { Level/ } \\
\text { points }\end{array}$ & 1 & 2 & 3 & 4 \\
\hline-300 & & & & -8 & & \multirow{8}{*}{ 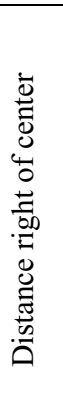 } & 800 & 302 & 217 & 174 & 184 \\
\hline-150 & & -4 & -3 & -10 & & & 700 & 279 & 217 & 137 & 147 \\
\hline 0 & & 13 & 6 & -8 & & & 600 & 277 & 213 & 127 & 152 \\
\hline 150 & 18 & 22 & 14 & -17 & & & 500 & 274 & 200 & 136 & 157 \\
\hline 300 & 24 & 67 & 64 & -18 & & & 400 & 269 & 187 & 157 & 157 \\
\hline 450 & 26 & 126 & 125 & -16 & & & 300 & 272 & 187 & 187 & 170 \\
\hline 600 & 27 & 151 & 133 & -18 & & & 200 & 267 & 214 & 174 & 142 \\
\hline 750 & 24 & 155 & 134 & -16 & & & 100 & 267 & 182 & 149 & 117 \\
\hline 900 & 25 & 154 & 135 & -13 & -18 & $\mathrm{C} / \mathrm{L}$ & 0 & 264 & 185 & 134 & 112 \\
\hline 1050 & 25 & 152 & 127 & -13 & -20 & \multirow{8}{*}{  } & 100 & 262 & 190 & 140 & 110 \\
\hline 1200 & 27 & 123 & 93 & -10 & -18 & & 200 & 259 & 195 & 145 & 112 \\
\hline 1350 & 25 & 121 & 96 & 28 & -16 & & 300 & 259 & 194 & 135 & 117 \\
\hline 1500 & 25 & 122 & 103 & 21 & -23 & & 400 & 256 & 192 & 126 & 131 \\
\hline 1650 & 23 & 129 & 111 & 10 & -25 & & 500 & 254 & 189 & 115 & 146 \\
\hline 1800 & 9 & 113 & 111 & -1 & -30 & & 600 & 252 & 188 & 136 & 182 \\
\hline 1950 & & 78 & 85 & -12 & -26 & & 700 & 255 & 189 & 182 & 226 \\
\hline 2100 & & & & -22 & -33 & & 800 & 266 & 235 & 237 & 270 \\
\hline 2250 & & & & -21 & -34 & & & & & & \\
\hline 2400 & & & & -27 & -38 & & & & & & \\
\hline 2550 & & & & -35 & -40 & & & & & & \\
\hline 2700 & & & & -41 & -41 & & & & & & \\
\hline 2850 & & & & -44 & & & & & & & \\
\hline
\end{tabular}


When calculating the energy consumption for the development of volumetric deformations over the entire damage zone (in the area from -300 to 2850), the result is $556,745 \mathrm{~J}$, which corresponds to a collision at a speed of $79 \mathrm{~km} / \mathrm{h}$ - this value is significantly different from the correct one, and therefore it is necessary to make calculations and determine the correct values of the stiffness coefficients. To begin with, we will limit the area of study of the obtained deformations to the contact spot - from 0 to $1,600 \mathrm{~mm}$, so the calculation will not take into account secondary deformations (where the deforming forces were not directly applied) [10]. Then we will bring the MDB bogie strain measurement system to a single one with the Jeep Grand Cherokee strain measurement system, and after calculating the energy costs and the speed equivalent to these costs, we will get the results shown in Table 2.

Table 2. Deformations and energy and velocity calculation results.

\begin{tabular}{|c|c|c|c|c|c|c|c|c|c|}
\hline & & & & Defo & ation & & & $\begin{array}{c}\text { Kinetic } \\
\text { energy, J }\end{array}$ & $\begin{array}{c}\text { Velocity, } \\
\mathrm{km} / \mathrm{h}\end{array}$ \\
\hline & & 0 & 300 & 600 & 900 & 1200 & 1500 & $E_{D}$ & V \\
\hline$\ddot{y}$ & 1 Level & - & 24 & 27 & 25 & 27 & 25 & 358472,72 & 63,48 \\
\hline 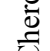 & 2 Level & 13 & 67 & 151 & 154 & 123 & 122 & 369410,14 & 64,44 \\
\hline $\bar{Z}$ & 3 Level & 6 & 64 & 133 & 135 & 93 & 103 & 368759,21 & 64,38 \\
\hline$\tilde{\sigma}$ & 4 Level & -8 & -18 & -18 & -13 & -10 & 21 & 485610,78 & 73,88 \\
\hline 巳্ & 5 Level & - & - & - & -18 & -18 & -23 & 497101,54 & 74,75 \\
\hline & Average & 4 & 34 & 73 & 57 & 43 & 50 & 364025,91 & 63,97 \\
\hline & 1 Level & 266 & 254 & 259 & 267 & 269 & 279 & & \\
\hline 合 & 2 Level & 235 & 189 & 195 & 182 & 187 & 217 & & \\
\hline$\Xi$ & 3 Level & 237 & 115 & 145 & 149 & 157 & 137 & & \\
\hline $\bar{\Sigma}$ & 4 Level & 270 & 146 & 112 & 117 & 157 & 147 & & \\
\hline & Average & 252 & 176 & 178 & 179 & 193 & 195 & & \\
\hline
\end{tabular}

Thus, it was found that when using the values of the penetration depth in the measurement area from 0 to $1600 \mathrm{~mm}$ at the levels of 1,2,3 and averaged, the results of the collision velocity calculations are quite accurate. Further calculations will use the averaged strain values of both the Jeep Grand Cherokee and the MDB truck.

To solve this scientific problem-differentiating the rigidity of the side of the car-it is necessary to divide it into 3 stages:

1) Divide the direct contact area into equal sections so that they coincide on the car and on the MDB trolleys;

2) Calculate the forces acting at the time of the collision for each section for the Jeep Grand Cherokee car and, based on the law of equality of action and reaction, determine that the calculated forces for the Jeep Grand Cherokee car are respectively equal to the forces on the MDB truck;

3) Set the differentiated stiffness coefficients for each section of the direct contact zone based on the obtained values of the acting forces.

At the first stage, it is logical to divide the zone of direct contact into sections, the rigidity inside of which is assumed to be homogeneous (the same), for example, a section of the body pillar, a section of the front door panel, etc., Thus taking into account the heterogeneity of the stiffness characteristics of the side of the car, which is the subject of research in this work $[11,12]$. 
According to the proposed method, it is necessary to calculate the impact forces acting on the sections of the MDB trolley (in the calculations, $b$ is denoted), for this the formula is used:

$$
F_{b i}=\frac{L_{b i}}{\cos \left(\alpha_{b}\right)} \times\left(A_{b i}+\frac{B_{b i}}{2} \times\left(C_{b i}+C_{b i+1}\right)\right)
$$

$F_{b i}$ - impact force on the $i$-th section (maximum) of the MDB truck;

$L_{b i}$ - length of the truck section MDB;

$i$ - contact spot section number;

$\alpha_{b}$ - the angle between the normal and the direction of the impact force;

$A_{b i}$ - the coefficient of rigidity of the MDB trolley, is equal to $35023.6 \mathrm{~N} / \mathrm{m}$;

$B_{b i}$ - the stiffness coefficient of the MDB trolley, equal to $517,049 \mathrm{~N} / \mathrm{m} 2$;

$C_{b i}, C_{b i+1}$ - values of deformations at the boundaries of the $i$-th section of the MDB trolley.

Also based on the law of equality of action and reaction:

$$
F_{b i}=F_{v i},
$$

$F_{v i}$ - the impact force on the i-th section (maximum) of the Jeep Grand Cherokee.

At this stage of calculations, it is necessary to clarify that to solve the problem of the presence of two unknowns in the equation (the stiffness coefficients $A$ and B for the Jeep Grand Cherokee), b0 and b1 are used -the permissible range of their values is known [13].

Using the set of b0v values for the Jeep Grand Cherokee, the blv values for each i-th section are calculated using the formula:

$$
b_{1 v i}=\frac{-b_{0 v i}+\sqrt{b_{0 v i}^{2}+\frac{2 \times 3.6^{2} \times L \times F_{i}}{L_{i} \times m_{v}} \times\left(C_{v i}+C_{v i+1}\right)}}{C_{v i}+C_{v i+1}},
$$

$b_{0 v i}$ - zero-strain speed on the $i$-th section of the Jeep Grand Cherokee

$L$ - width of the direct contact area;

$L_{i}$ - width of the $i$-th section;

$F_{i}$ - impact force on the $i$-th section (maximum);

$m_{v}$ - weight of the Jeep Grand Cherokee;

$C_{v i}, C_{v i+1}$-values of deformations at the boundaries of the $i$-th section of the Jeep Grand Cherokee.

As a result, it becomes possible to determine the differentiated stiffness coefficients of the side of the Jeep Grand Cherokee car at each i-th section of the direct contact zone using the formulas:

$$
\begin{aligned}
& A_{v i}=\frac{m_{v} \times b_{0 v i} \times b_{1 v i}}{3.6^{2} \times L}, \\
& B_{v i}=\frac{m_{v} \times b_{1 v i}^{2}}{3.6^{2} \times L},
\end{aligned}
$$

Thus, we apply the proposed method to the studied road accident and using matrix algebra, we get the values of deformations at the boundaries of the sections and their lengths as vectors: 


$$
\begin{aligned}
& C_{b}=\left(\begin{array}{l}
0.252 \\
0.176 \\
0.178 \\
0.179 \\
0.193 \\
0.195
\end{array}\right) ; \\
& C_{v}=\left(\begin{array}{l}
0.003667 \\
0.03425 \\
0.07325 \\
0.0566 \\
0.043 \\
0.0496
\end{array}\right) ; \\
& L_{b} \doteqdot L_{v} ;
\end{aligned}
$$

The total peak forces at each i-th section of the direct contact zone on the MDB trolley are then set:

$$
\begin{gathered}
F_{b}=\frac{L_{b i}}{\cos \left(\alpha_{b}\right)} \times\left(A_{b i}+\frac{B_{b i}}{2} \times\left(C_{b i}+C_{b i+1}\right)\right)=\left(\begin{array}{l}
44326.78 \\
38485.77 \\
38702.11 \\
39862.44 \\
41140.78
\end{array}\right) ; \\
\sum F_{b}=202517,89 ;
\end{gathered}
$$

As can be seen from the results obtained, the force value is maximum in the first section, then there is a decrease and again an increase in the value of the deforming force in the 5th section-this is due to the structural rigidity of the side of the car body: in the 1st section, the impact falls on the area of the front pillar of the car, then "spreads" over the front door, the area of which, as is known, is less resistant to deformations arising under the influence of external forces, and then again in the section 5, the impact is applied to the car pillar (medium), where the body stiffness is higher than in the area of the front (and rear) doors.

Having obtained the values of the maximum deforming forces on each section of the MDB trolley (and as mentioned above, according to the law of equality of action and reaction, these forces are equal on the corresponding sections of the Jeep Grand Cherokee car), it is possible to determine the proportion of the dispersion of the reduced speed to the area of the contact zone (b1v) on the i-th section of the side of the car under study-a participant in a staged accident.

$$
b_{1 v}=\frac{-b_{0 v i}+\sqrt{b_{0 v i}^{2}+\frac{2 \times 3.6^{2} \times L \times F_{i}}{L_{i} \times m_{v}} \times\left(C_{v i}+C_{v i+1}\right)}}{C_{v i}+C_{v i+1}}=\left(\begin{array}{l}
170.01 \\
108.5 \\
101.3 \\
114.1 \\
119.5
\end{array}\right) ;
$$

As can be seen from the results of calculations blv-the rigidity of the car body in the area of the front pillar is significantly greater than the rigidity of the door panel.

Using all the results of the calculations, it becomes possible to solve the scientific problem - to differentiate the rigidity of the structure of the side of the car in the area of direct contact: 


$$
\begin{gathered}
A_{v i}=\frac{m_{v} \times b_{0 v i} \times b_{1 v i}}{3.6^{2} \times L}=\left(\begin{array}{l}
80669.2 \\
51465.4 \\
48086.8 \\
54127.2 \\
56719.9
\end{array}\right) ; \\
B_{v i}=\frac{m_{v} \times b_{1 v i}^{2}}{3.6^{2} \times L}=\left(\begin{array}{l}
3428724 \\
1395557 \\
1218340 \\
1543647 \\
1695073
\end{array}\right) ;
\end{gathered}
$$

Thus, the stiffness coefficients were set on different sections of the side of the Jeep Grand Cherokee. To verify and confirm the correctness of their values, it is necessary to determine the maximum forces acting on each i-th section and compare the results with the values of the deforming forces on the corresponding sections of the MDB trolley:

$$
\begin{gathered}
F_{v}=\frac{L_{v i}}{\cos \left(\alpha_{v}\right)} \times\left(A_{v i}+\frac{B_{v i}}{2} \times\left(C_{v i}+C_{v i+1}\right)\right)=\left(\begin{array}{l}
44326.78 \\
38485.77 \\
38702.11 \\
39862.44 \\
41140.78
\end{array}\right) ; \\
\sum F_{v}=\sum F_{b}=202517,89 .
\end{gathered}
$$

As a result, it was found that the peak values of the deforming forces on each i-th section of the car and truck, as well as their total values, exactly coincide, which means that the law of equality of action and reaction is fulfilled, thereby confirming the accuracy of calculations and the correctness of the results of the proposed method for determining the differentiated rigidity of the car body.

It should be noted that this algorithm can also be used when calculating the coefficients of not only the side of vehicles, but also the front and rear, but the following limitations should be taken into account when conducting studies based on the presented methodology:

1) The stiffness coefficients of the striking object (truck or car) are known

2) The values of deformations of both vehicles-participants of the accident, as well as the zones of their direct contact and the zones of secondary deformations are established

3) The direct contact areas on both vehicles must be the same width and can be divided into equal (corresponding) contact areas.

As it was established in this work, the rigidity of the side of the car is not uniform in its width. In the case of calculations based on averaged coefficients, the lack of energy for the stiffer part (the rack area) is compensated by the excess for the more fragile areas (the door), and as a result, the calculation of the kinetic energy required to produce deformations will be inaccurate.

However, if, as a result of a road traffic situation, the car was damaged only in the area of the side pillars, the result of calculating the kinetic energy spent on the deformation of the car, according to the average stiffness coefficients, will be higher than the actual one, and vice versa, in a situation where the deformations of the car involved in the accident only in the area of the doors, the result of the calculation with the average stiffness coefficients will be overestimated $[14,15]$. 
Therefore, the presented method of differentiating the rigidity of the side part of the car is necessary for automotive experts to obtain unambiguous answers to the questions raised.

In the foreseeable future, it seems appropriate to differentiate the rigidity of the side of the car in height, then simultaneously in width and height, and to develop a methodology that allows you to use an unequal measurement step when measuring the depth of penetration this will avoid restrictions and increase the accuracy of the results in the production of road transport expertise.

\section{References}

1. V.L. Mitunyavichus, Estimation of strain energy in collision analysis, P. 24

2. E. Kurakina, S. Evtiukov, G. Ginzburg, Systemic indicators of road infrastructure at accident clusters, Architecture and Engineering, 5 (1), p. 51-58 (2020)

3. E.E. Medres, E.V. Golov, T.I. Babenko, Factors affecting the uniformity of road transport movement in conditions of saturated traffic flows, Transport Business of Russia, 2 (2017)

4. P. Rajczyk, S. Evtiukov, New technological solutions in improving road safety, Transportation research procedia (2018)

5. E. Kurakina, S. Evtiukov, J. Rajczyk, Forecasting of road accident in the dvre system, Transportation research procedia, (2018)

6. US Department of Transportation. CRASH 3 Technical Manual. Cambridge: NHTSA, P. 458 (1986)

7. S.A. Evtyukov, Ya.V. Vasiliev, Handbook on the examination of road accidents, St. Petersburg, P. 512 (2015)

8. S. Evtiukov, E. Golov, G. Ginzburg, Finite element method for reconstruction of road traffic accidents, Transportation research procedia, (2018)

9. https://www.nhtsa.gov/

10. V.N. Dobromirov, S.S. Evtyukov, E.V. Golov, Modern technologies of primary inspection of a road accident site, Bulletin of Civil Engineers, № 2(61), (2017)

11. https://pravorub.ru/articles/caseadm/auto_disputes/

12. V.N. Nikonov, Reconstruction of the circumstances of an accident: An introduction to modern methods of expert research, Using crash tests» Publishing Solutions, P. 126 2017

13. S.S. Evtyukov, E.V. Golov, The choice of coefficients in determining the cost of kinetic energy for the deformation of the car, Bulletin of Civil Engineers, 1 (72) (2019)

14. E.V. Golov, S.S. Evtyukov, Ya.V. Vasiliev, Differentiation of the rigidity of the front part of the car, In the collection: Information technologies and innovations in transport. Proceedings of the VI International Scientific and Practical Conference, pp. 293-299 (2020)

15. S. Evtyukov, E. Golov, J. Rajczyk, Improving the accuracy of stiffness coefficient calculation when estimating the kinetic energy spent on vehicle deformation, Architecture and engineering, 1 (2020) 